\title{
Combined endoscopic laser therapy and brachytherapy for palliation of oesophageal carcinoma: a pilot study
}

\author{
P Renwick, V Whitton, K Moghissi
}

\begin{abstract}
Palliative treatment for oesophageal malignancy aims to maximise symptom relief with minimal disturbance to the patient. Twenty one patients with oesophageal carcinoma were studied prospectively to assess the combined efficacy of laser and brachytherapy in the palliation of oesophageal carcinoma, 20 were unsuitable for resectional surgery because of tumour extent and one patient underwent the treatment protocol after myocardial infarction, for symptom relief before resection. Two patients died at hospital and the remaining 19 survived from 9 to 455 days (mean 140 days). All patients tolerated the procedure well and improvement in swallowing was noted in 19 who survived the procedure - an improvement that was maintained until their death. However, five patients required oesophageal dilatation after the initial treatment. Results were not affected by the histology of the tumour. In summary, combined endoscopic laser and brachytherapy is effective palliation for oesophageal carcinoma and may be particularly appropriate in those patients with cervical and upper thoracic tumours in whom intubation may be unsatisfactory.
\end{abstract}

Many patients with carcinoma of the oesophagus have advanced disease at presentation. Resectability rates vary widely from centre to centre, ${ }^{1-3}$ though an extensive review by Earlam and Cunha-Melo ${ }^{4}$ reports a rate of $40 \%$, which is confirmed by Watson. ${ }^{5}$ The main aim of treat-

TABLE I Swallowing pattern of patients before procedure

Humberside Cardiothoracic Surgical Centre, Castle Hill Hospital, Hull P Renwick

K Moghissi

Radiation Physics

Department, Princess

Royal Hospital, Hull

V Whitton

Correspondence to:

Dr P Renwick, Cardio-

thoracic Surgical Centre

Castle Hill Hospital

Cottingham, Hull, $\mathbf{N}$

Humberside HU16 $5 \mathrm{JQ}$

Accepted for publication

27 July 1991

\begin{tabular}{lc}
\hline Dysphagia scale & $\begin{array}{l}\text { No of patients } \\
\text { before treatment }\end{array}$ \\
\hline 0 (No dysphagia) & 0 \\
1 (Dysphagia to solids) & 0 \\
2 (Dysphagia to semisolids) & 11 \\
3 (Dysphagia to soft/puree) & 6 \\
4 (Total dysphagia) & 4 \\
\hline
\end{tabular}

TABLE II Anatomical distribution and histology of lesions

\begin{tabular}{lrl}
\hline Location of lesion & No & Histology; type of carcinoma \\
\hline Upper/cervical & 3 & Squamous (2); undifferentiated (1) \\
Mid & 11 & Squamous (8); adeno (3) \\
Lower & 7 & Adeno (7) \\
\hline
\end{tabular}

ment in most patients therefore is palliation of symptoms and improvement in the quality of their remaining life. Many techniques have been used for palliation, and since the availability of Nd Yag laser in clinical practice, endoscopic laser therapy has been effectively employed in the relief of dysphagia. There has also been a recent resurgence of interest in intraluminal radiotherapy.

As far as we are aware there has been only one previous report on the combined effect of laser and brachytherapy. ${ }^{6}$ We report the findings of a pilot study on the combined effectiveness of endoscopic laser and brachytherapy in the palliation of oesophageal cancer.

\section{Patients and methods}

\section{PATIENTS}

Over an 18 month period, 21 patients (nine men and 12 women) underwent combined laser and brachytherapy for malignant dysphagia. Their age at presentation was between 46 and 82 years (mean 69 years). All patients presented with severe dysphagia (grade 2-4 according to our classification), ${ }^{7}$ and as shown in Table I, 12 patients had associated weight loss of between 7 and $10 \%$ of their usual weight.

Twenty of the 21 patients were unsuitable for surgical resection; the remaining patient presented with a resectable carcinoma but suffered a preoperative myocardial infarction with superimposed Gram negative septicaemia. He underwent the procedure to allow adequate nutrition before elective resectional surgery two months later. All patients had histologically proved malignancy and underwent preoperative assessment by barium swallow, abdominal ultrasound, and endoscopy. The anatomical tumour distribution and histological breakdown are shown in Table II.

\section{EQUIPMENT AND METHOD}

Both laser application and initial placement of the brachytherapy tube were carried out through an open ended rigid oesophagoscope.

The laser equipment consisted of the $100 \mathrm{~W}$ Nd Yag laser (Fibrelase 100, Pilkington Medical Systems Ltd, Glasgow), the laser energy being delivered through a single flexible fibre with a 

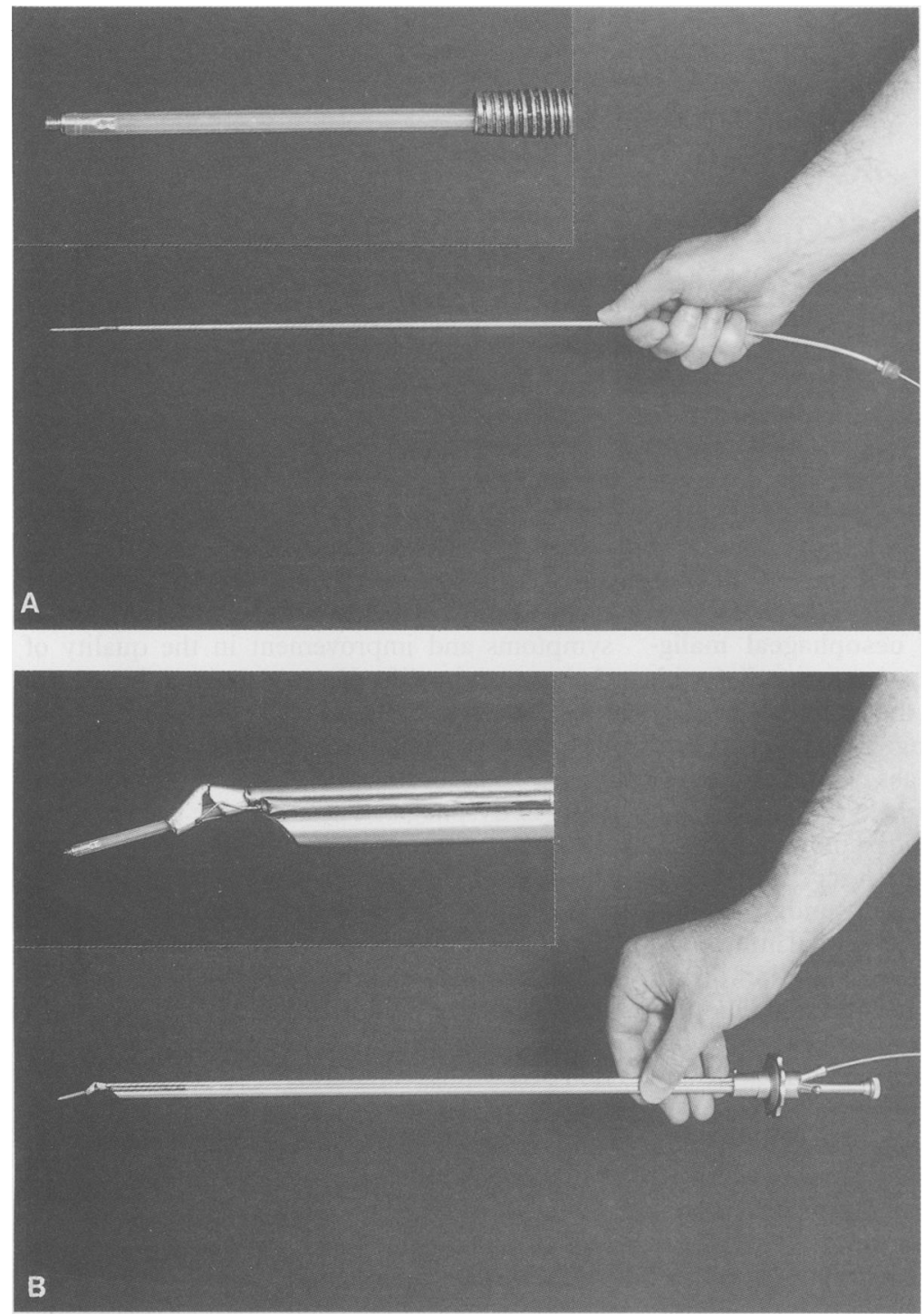

Figure 1 (A): Metal laser applicator with fibre. (B) Moghissi-fessop laser endoscope with fibre in situ. to the remote afterloading unit (Nucletron International BV type selectron LDR) is located. These units allow staff exposure to be virtually eliminated as the radioactive material is transferred automatically under computer control. At all times, patients are monitored by closed circuit television from an adjacent control area outside the treatment room. A source carrier is inserted into the applicator and the unit programmed and started. The prescribed dose was 15.0 Gray to the centre of the tumour, $1 \mathrm{~cm}$ from the axis of the applicator. Normally, all 48 caesium 137 beadsources were used over $12 \mathrm{~cm}$ for about one hour.

All patients returned to the thoracic surgical wards for follow up care. A routine chest and lateral neck radiograph was performed after the procedure to exclude perforation. If this was satisfactory, clear fluids were begun six hours after the procedure and a normal diet instituted 24 hours later. A barium swallow was performed before discharge home. The patients were followed up in the outpatient clinic regularly during their survival.

\section{Results}

There were two hospital deaths during this study. Both patients had disseminated disease at the time of treatment. One patient developed bronchopneumonia, presumed secondary to aspiration, and the other suffered a fatal myocardial infarction, unrelated to treatment, on the third day after treatment. Both patients underwent necropsy examination. All the 19 surviving patients achieved good relief of their dysphagia, and were discharged home on a solid or semisolid diet. Figure 2 shows the change in the dysphagia grade of these patients after treatment. Survival times range from 9-455 days, with an average survival period of 140 days to date. Five patients are still alive $3,9,24,59$, and 60 weeks after the procedure (Table III).

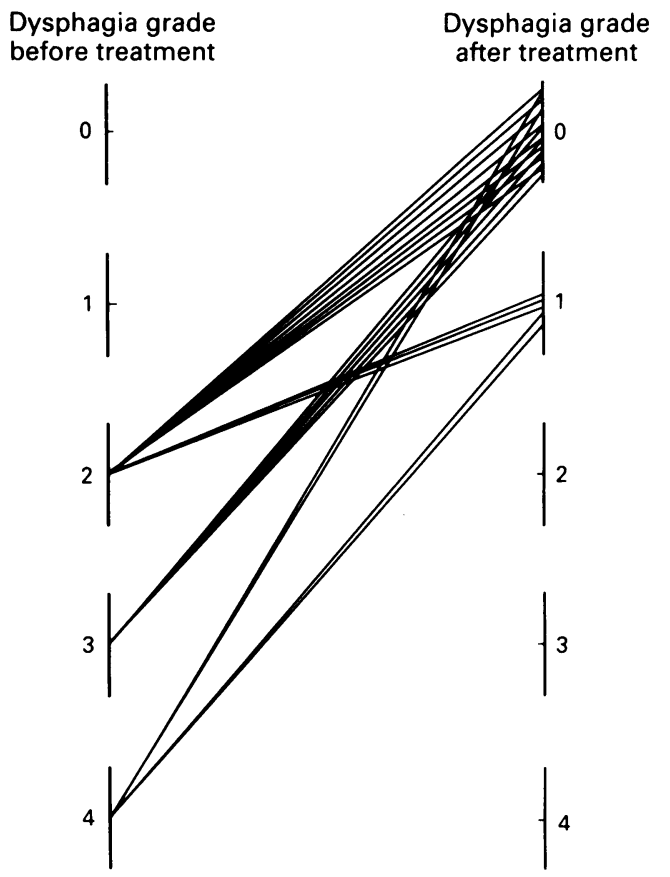

Figure 2: Change in dysphagia scale in 19 patients after treatment by laser and brachvtherapy. separate helium neon aiming light for precise targeting via the metal laser applicator. This applicator, devised in the department, is essentially a fine metal tube accommodating the fibre used alone (Fig 1A) or in conjunction with the endoscope (Fig 1B). The laser was used in its non-contact mode employing multiple pulses of $40-60 \mathrm{~W}$ for a duration of $8-10$ seconds.

The oesophagoscopy, laser therapy, and insertion of brachytherapy tube were performed under general anaesthesia with endotracheal intubation. The oesophagoscope was passed first, the site of lesion carefully noted and, in most cases, bouginage carried out. Laser radiation was performed next using the non-contact mode in a prograde fashion. The brachytherapy applicator with dummy source train was then introduced and placed in position through the tumour. Once the position of the tube was checked by radiography and found to be satisfactory, it was then immobilised at the mouth by means of a simple mask.

At this stage the patient was allowed to recover from the anaesthetic and to breathe spontaneously before being transferred to the radiotherapy centre, where a source carrier connected 
TABLE III Patient outcome

\begin{tabular}{|c|c|c|c|c|}
\hline Patient No & Histology & $\begin{array}{l}\text { Tumour length } \\
(\mathrm{cm})\end{array}$ & Location & Survival time (days) \\
\hline 1 & Squamous & Total & & 112 \\
\hline 2 & Large Cell (Undiff) & $3-4$ & Upper $1 / 3$ rd & 18 \\
\hline 3 & Squamous & $3-5$ & Mid 1/3rd & 2 (in hospital death) \\
\hline 4 & $\begin{array}{l}\text { Adeno } \\
\text { Ad }\end{array}$ & $3-4$ & Lower $1 / 3$ rd & 455 \\
\hline 5 & Squamous & $3-4$ & Mid $1 / 3$ rd & 42 \\
\hline 6 & Adeno & $3-4$ & Lower $1 / 3$ rd & $410^{\star}$ \\
\hline 7 & Adeno & $3-4$ & Mid $1 / 3$ rd & 50 \\
\hline 8 & Adeno & $4-5$ & Lower $1 / 3$ rd & $404^{\star}$ \\
\hline 9 & Squamous & 5 & Upper $1 / 3$ rd & 51 \\
\hline 10 & Squamous & $3-4$ & Lower $1 / 3$ rd & 5 (in hospital death) \\
\hline 11 & Squamous & $6-8$ & $\mathrm{Mid} /$ Low $1 / 3$ rd & 9 \\
\hline 12 & Squamous & $3-4$ & Lower $1 / 3$ rd & 312 \\
\hline 13 & Adeno & $3-4$ & Lower $1 / 3$ rd & 77 \\
\hline 14 & Adeno & $3-4$ & Mid $1 / 3 r d$ & $19^{\star}$ \\
\hline 15 & Adeno & $5-6$ & Lower $1 / 3$ rd & 99 \\
\hline 16 & Squamous & $4-5$ & Mid $1 / 3$ rd & 139 \\
\hline 17 & Squamous & $4-5$ & $\operatorname{Mid} 1 / 3$ rd & 54 \\
\hline 18 & Adeno & $3-4$ & Lower $1 / 3$ rd & 80 \\
\hline 19 & Squamous & $7-8$ & Mid $1 / 3$ rd & 117 \\
\hline 20 & Adeno & $6-7$ & Mid $1 / 3$ rd & $63^{\star}$ \\
\hline 21 & Adeno & $3-4$ & Lower $1 / 3$ rd & $166^{\star}$ \\
\hline
\end{tabular}

^ Denotes still alive at time of publication.

Symptom relief does not seem to be related to the level of presentation (upper margin) or attendant histology of the oesophageal malignancy, although generally longer survival times are seen in mid-oesophageal and lower oesophageal lesions. The patient who underwent treatment for symptom relief before surgical resection remains alive and well at 16 months after the procedure. All patients tolerated the treatment well with none failing to complete the course of radiotherapy. Five patients required subsequent dilatation at between 6 and 44 weeks after the procedure. Four of these patients were noted at that time to have no visual or histological evidence of tumour recurrence, though a fibrous stricture was present. Three of these patients required repeat dilatation at 14,16 , and 24 weeks after the procedure with recurrent tumour present in two on histology.

\section{Discussion}

Over the years various techniques for palliative treatment of oesophageal carcinoma have evolved, each with its own advantages and drawbacks. Probably the best form of palliation, if the patient is fit enough, is resectional surgery in the hands of a committed oesophageal surgeon. ${ }^{8}$ Palliative intubation of the oesophagus may be performed either surgically or endoscopically, with the latter achieving lower mortality rates. ${ }^{59}$ The quality of symptom relief is, however, challenged as most patients can tolerate only soft or liquid diets and a significant number incur further dysphagia as a result of tube dysfunction. ${ }^{10}$ Moreover general experience shows that indwelling oesophageal tubes are not well tolerated in high oesophageal cancer.

Radiotherapy may be delivered either externally, ${ }^{11}$ with only slow relief of dysphagia, or internally with minimal morbidity and a good rate of relief of dysphagia. ${ }^{12}$ Repeated bouginage becomes an increasing burden and increases in difficulty as the disease progresses. The role of chemotherapy in the palliation of oesophageal cancer has not as yet been truly evaluated and, as a final resort, gastrostomy feeding may be established but this is not acceptable to many patients.

In 1982 Fleischer ${ }^{13}$ first reported the effective use of $\mathrm{Nd}$ Yag laser in the relief of malignant dysphagia with the results being confirmed by other groups. ${ }^{14-16}$

The rationale of combined laser and brachytherapy is that laser therapy debulks intraluminal tumour while brachytherapy aims to treat mural invasion, thus aiming therapy at different levels unobtainable by single modality treatment.

The results of this pilot study indicate that combined laser and brachytherapy provide useful palliative results with most patients achieving normal or near normal swallowing, usually within a single treatment session. All patients are reviewed regularly as outpatients and are admitted for dilatation and follow up oesophagoscopy if their swallowing deteriorates so that they cannot manage semisolid food. Patients and general practitioners are offered an open review service if they are unhappy with the swallowing status.

In our patients we have applied laser in its noncontact mode through a rigid open ended oesophagoscope. We believe that for this combined procedure the rigid instrument has the following advantages over its flexible counterpart:

(a) It allows good suction of fluid and oesophageal debris which would complicate the treatment;

(b) It allows good visualisation of the oesophageal lumen and intraluminal tumour projection;

(c) It permits prograde laser fulguration and smoke aspiration and gradual dilatation in tight obstructions. We do however use the flexible endoscope in conjunction with the rigid system in patients with less tight stenoses and smaller tumour lengths.

One unexpected but interesting finding of this treatment protocol is that laser and brachytherapy may be useful for symptom palliation in patients requiring prolonged investigation before resective surgery, as was the case in one of our patients.

We believe that when palliation is required in oesophageal carcinoma the clinician should be aware of a range of available treatments in order to select the most appropriate. For instance, intubation of the oesophagus may be suitable for lower oesophageal lesions but it is not well tolerated in the upper oesophagus. On the other hand laser and brachytherapy, individually or together, are suitable in all cases including upper oesophageal cancer. This study also shows that excellent symptom palliation can be achieved at a single treatment session thus reducing patient stay in hospital to a minimum. This differs from the protocol of Bader $e t a l^{6}$ where several laser sessions were followed one week later by endoluminal radiotherapy at one to two weekly intervals. It is interesting that repeat endoluminal radiotherapy does not seem to increase complications in the study by Bader. However, we have not used repeat endoluminal radiotherapy to date.

This study reinforces our initial opinions that combined laser and brachytherapy is useful in the palliation of malignant dysphagia and will continue to be used for patients with unresectable oesophageal carcinoma in our department, 
although this implies a previous healthy working knowledge of upper alimentary endoscopy of both rigid and flexible formats.

The authors thank Dr R A Dealey for his advice and management with regard to the brachytherapy, Mrs C Dixon for help with the preparation of the manuscript, and Mrs $M$ Dench for help in typing the manuscript.

1 Salama FD, Leong YP. Resection for carcinoma of the oesophagus. F $R$ Coll Surg Edinb 1989; 34: 97-100.

2 Moghissi K. Essentials of thoracic and cardiac surgery. London: Heinemann, 1986.

3 Cotton MH, Sammon AM. Carcinoma of the oesophagus in Transkei: treatment by intubation. Thorax $1989 ; 44: 42-7$.

4 Earlam R, Cunha-Melo JR. Oesophageal squamous cell carcinoma: A critical review of surgery. Br $\mathcal{F}$ Surg 1980; 67: 381-90.

5 Watson $\dot{A}$. A study of the quality and duration of survival following resection, endoscopic intubation and surgical intubation in oesophageal carcinoma. Br f Surg 1982; 69: 585-8.

6 Bader M, Dittler HJ, Ultsch B, Ries G, Siewert JR. Palliative treatment of malignant stenoses of the upper gastrointestinal tract using a combination of laser and afterloading therapy. Endoscopy 1986; 18 (suppl 1): 27-31.
7 Moghissi K, Hornshaw J, Teasdale PR, Dawes EA. Parenteral nutrition in carcinoma of the oesophagus treated by surgery: nitrogen balance and clinical studies. Br $\mathcal{F}$ Surg 1977; 64: 125-8.

8 Bancewicz J. Cancer of the oesophagus. BMF 1990; 300: 3 9 Ogilvie AL, Dronfield MW, Ferguson R, Atkinson M Palliative intubation of oesophagogastric neoplasms at Palliative intubation of oesophagogastric

10 Pffleiderer AG, Goodall D, Holmes GKT. The consequences and effectiveness of intubation in the palliation of dysphagia and effectiveness of intubation in the paliation of dysphagia f Surg 1982; 69: 365-8.

11 Earlam R, Cunha-Melo JR. Oesophageal squamous cell carcinoma. II: A critical review of radiotherapy. BrF Surg 1980; 67: 457-61.

12 Giles Rowland C, Pagliero KM. Intracavitatory irradiation in palliation of carcinoma of the oesophagus and cardia. Lancet 1985; ii: 981-2.

13 Fleischer D, Kessler F. Endoscopic Nd Yag laser therapy for carcinoma of the oesophagus: A new form of palliative treatment. Gastroenterology 1983; 85: 600-6.

14 Bown SG, Hawes R, Matthewson K, Swain CP, Barr H Boulos $\mathrm{PB}$, et al. Endoscopic laser palliation for advanced malignant dysphagia. Gut 1987; 28: 799-807.

15 Krasner N, Barr H, Skidmore C, Morris AI. Palliative laser therapy for malignant dysphagia. Gut 1987; 28: 792-8.

16 Moon BC, Woolfson IK, Mercer CD. Nd Yag laser vaporisation for palliation of obstructing esophageal carcinomas. $\mathcal{f}$ Thorac Cardiovasc Surg 1989; 98: 11-5. 